\title{
THE NEHARI MANIFOLD APPROACH FOR DIRICHLET PROBLEM INVOLVING THE $p(x)$-LAPLACIAN EQUATION
}

\author{
Rabil A. Mashiyev, Sezai Ogras, Zehra Yucedag, and Mustafa Avci
}

\begin{abstract}
In this paper, using the Nehari manifold approach and some variational techniques, we discuss the multiplicity of positive solutions for the $p(x)$-Laplacian problems with non-negative weight functions and prove that an elliptic equation has at least two positive solutions.
\end{abstract}

\section{Introduction}

In this paper, we study the multiplicity of positive solutions for the following elliptic equation

$\left(E_{\lambda}\right) \quad\left\{\begin{array}{cc}-\Delta_{p(x)} u(x)=\lambda a(x)|u|^{q(x)-2} u+b(x)|u|^{h(x)-2} u & \text { in } \Omega \\ u(x)=0 & \text { on } \partial \Omega,\end{array}\right.$

where the following conditions are satisfied:

$\Omega$ is a bounded domain with smooth boundary in $\mathbb{R}^{N}, N \geq 2, q, p, h \in C(\bar{\Omega})$ such that $1<q(x)<p(x)<h(x)<p^{*}(x)\left(p^{*}(x)=\frac{N p(x)}{N-p(x)}\right.$ if $N>p(x)$, $p^{*}(x)=\infty$ if $\left.N \leq p(x)\right), 1<p^{-}:=\operatorname{ess} \inf _{x \in \Omega} p(x) \leq p(x) \leq p^{+}:=\underset{x \in \Omega}{\operatorname{ess} \sup } p(x)<$ $\infty, 1<q^{-} \leq q^{+}<p^{-} \leq p^{+}<h^{-} \leq h^{+}, \lambda>0 \in \mathbb{R}$ and $a, b \in C(\bar{\Omega})$ are non-negative weight functions with compact support in $\Omega$.

Over the last decade, the variable exponent Lebesgue spaces $L^{p(x)}$ and the corresponding Sobolev space $W^{1, p(x)}$ have been a subject of active research area (we refer to $[8,11,12,18,19]$ for the fundamental properties of these spaces). These investigations are stimulated mainly by the development of the studies of problems in Elasticity, Electrorheological fluids, Image Processing, Flow in Porous Media, Calculus of Variations, Differential Equations with $p(x)$-growth (see Acerbi and Mingione [1], Diening [7], Buhrii and Mashiyev [5], Halsey [15], Mihăilescu and Rădulescu [21], Růžička [24], Zhikov [28]). Among these problems, the study of $p(x)$-Laplacian problems via variational methods is an

Received October 7, 2008; Revised November 4, 2008.

2000 Mathematics Subject Classification. 35B38, 35D05, 35J60, 35J70, 58E05.

Key words and phrases. variable exponent Lebesgue-Sobolev spaces, $p(x)$-Laplacian, variational methods, Nehari manifold, multiple positive solutions.

This Research Project was supported by DUAPK-2008-59-74, Dicle University, Turkey. 
interesting topic. A lot of researchers have devoted their works to this area (see Chabrowski and Fu [6], Fan [10], Fan and Zhang [13, 14], Mihăilescu [21], Mihăilescu and Rădulescu [22], Harjulehto, Hästö, Koskenoja and S. Varonen [16], Hästö [17], Ogras, Mashiyev, Avci and Yucedag [23]). We refer to the $p(x)$-Laplace operator $\Delta_{p(x)} u:=\operatorname{div}\left(|\nabla u|^{p(x)-2} \nabla u\right)$, where $p$ is a continuous non-constant function. This differential operator is a natural generalization of the $p$-Laplace operator $\Delta_{p} u:=\operatorname{div}\left(|\nabla u|^{p-2} \nabla u\right)$, where $p>1$ is a real constant. However, the $p(x)$-Laplace operator possesses more complicated nonlinearity than $p$-Laplace operator, due to the fact that $\Delta_{p(x)}$ is not homogeneous. This fact implies some difficulties; for example, we can not use the Lagrange Multiplier Theorem in many problems involving this operator.

In recent years, the similar problems of the form $\left(E_{\lambda}\right)$ have been studied by many authors using various methods. In [20] for the case $p(x)>1$ and $1<q<\inf _{\Omega} p<\sup _{\Omega} p<h<\min \left\{N, \frac{N \cdot p^{-}}{N-p^{-}}\right\}$and $a(x) \equiv a, b(x) \equiv$ $b>0$, using Ekeland's variational principle and the mountain-pass lemma Mihăilescu proved that, if $a$ and $b$ small enough then there are two distinct solutions for the problem; in [22] under the assumptions $1<\min _{x \in \bar{\Omega}} q(x)<$ $\min _{x \in \bar{\Omega}} p(x)<\max _{x \in \bar{\Omega}} q(x)$, where $p(x), q(x)$ are continuous on $\bar{\Omega}, h(x)=0$, $a(x) \equiv 1, b(x)=0$, Mihăilescu and Rădulescu showed that there exists $\lambda^{*}$ such that any $\lambda \in\left(0, \lambda^{*}\right)$ is an eigenvalue for the problem by using Ekeland's variational principle and the mountain-pass lemma; in [14] for the case $p(x)=q(x)>1, h(x)=0$, where $p(x)$ is continuous on $\bar{\Omega}$, and $a(x) \equiv$ $1, b(x)=0$, Fan, Zhang, and Zhao obtained that, $\Lambda=\Lambda_{p(x)}$, the set of eigenvalues, is a nonempty infinite set such that $\sup \Lambda=+\infty$. In addition, they present some sufficient conditions for inf $\Lambda=0$ and for inf $\Lambda>0$, respectively; in [4] under the conditions $p(x)=2, q(x)=2$ and $1<h<\frac{N+2}{N-2}$, where $h$ is constant, and $a(x), b(x): \bar{\Omega} \subset \mathbb{R}^{N} \rightarrow \mathbb{R}$ are smooth functions which may change sign in $\bar{\Omega}$, Brown and Zhang used the relationship between the Nehari manifold and fibrering maps to show how existence and nonexistence results for positive solutions of the equation are linked to properties of the Nehari manifold; in [2] Afrouzi, Mahdavi, and Naghizadeh dealt with the similar problem for the case $p(x)=q(x)=p, h(x)=h, 1<h<p$, $a(x)=1$ and $b(x): \Omega \subset \mathbb{R}^{N} \rightarrow \mathbb{R}$ is a smooth function which may change sign and they discussed the existence and multiplicity of non-negative solutions of the problem from a variational viewpoint by making use of the Nehari manifold. Under the conditions $p(x)=p, q(x)=q, h(x)=h$, $1<q<p<h<p^{*}\left(p^{*}=\frac{N p}{N-p}\right.$ if $N>p, p^{*}=\infty$ if $\left.N \leq p\right)$ and the weight functions $a(x) \equiv b(x) \equiv 1$, the authors Ambrosetti-Brezis-Cerami [3] have investigated equation $\left(E_{\lambda}\right)$. They found that there exists $\lambda_{0}$ such that equation $\left(E_{\lambda}\right)$ admits at least two positive solutions for $\lambda \in\left(0, \lambda_{0}\right)$, has a positive solution for $\lambda=\lambda_{0}$ and no positive solution exists for $\lambda>\lambda_{0}$, and also in [26] under 
the same assumptions and with sign-changing weight functions $a(x)$ and $b(x)$, Wu gave a variational proof of the existence of at least two positive solutions of equation $\left(E_{\lambda}\right)$ for $p \in\left(1, p^{*}\right)$, using Palais-Smale and decomposition of the Nehari manifold.

In this paper, we have generalized the articles of Ambrosetti-Brezis-Cerami $[3]$ and $\mathrm{Wu}[26]$, to the $p(x)$-Laplacian by using the Nehari manifold under the similar conditions. We shall discuss the multiplicity of positive solutions for the problem $\left(E_{\lambda}\right)$ and prove the existence of at least two positive solutions.

If we consider all above mentioned papers use of the Nehari manifold approach for the case $p(x)$-growth condition makes our study quite different and very interesting.

\section{Notations and preliminaries}

We will discuss our problem $\left(E_{\lambda}\right)$ in the variable exponent Sobolev space $W_{0}^{1, p(x)}(\Omega)$, so we need some theories and basic properties on spaces $L^{p(x)}(\Omega)$ and $W^{1, p(x)}(\Omega)$.

Write

$$
L_{+}^{\infty}(\Omega)=\left\{p \in L^{\infty}(\Omega): p^{-}>1\right\}
$$

Let's define by $\mathcal{U}(\Omega)$ the set of all measurable real functions defined on $\Omega$. For any $p \in L_{+}^{\infty}(\Omega)$, we denote the variable exponent Lebesgue space by

$$
L^{p(x)}(\Omega)=\left\{u \in \mathcal{U}(\Omega): \int_{\Omega}|u(x)|^{p(x)} d x<\infty\right\},
$$

which is equipped with the norm, so-called Luxemburg norm $[11,12,18]$

$$
|u|_{p(x)}=\inf \left\{\delta>0: \int_{\Omega}\left|\frac{u(x)}{\delta}\right|^{p(x)} d x \leq 1\right\}
$$

and $\left(L^{p(x)}(\Omega),|\cdot|_{p(x)}\right)$ becomes a Banach space, we call it as variable exponent Lebesgue space.

Let $c$ is a measurable real-valued function and $c(x)>0$ for $x \in \Omega$. Then the weighted variable exponent Lebesgue space $L_{c(x)}^{p(x)}(\Omega)$ is defined by

$$
L_{c(x)}^{p(x)}(\Omega)=\left\{u \in \mathcal{U}(\Omega): \int_{\Omega} c(x)|u(x)|^{p(x)} d x<\infty ; c(x)>0\right\}
$$

which is equipped with the norm

$$
|u|_{(p(x), c(x))}=\inf \left\{\delta>0: \int_{\Omega} c(x)\left|\frac{u(x)}{\delta}\right|^{p(x)} d x \leq 1\right\} .
$$


Proposition 2.1 ([11, 18]). The conjugate space of $L^{p(x)}(\Omega)$ is $L^{p^{\prime}(x)}(\Omega)$, where $\frac{1}{p^{\prime}(x)}+\frac{1}{p(x)}=1$. For any $u \in L^{p(x)}(\Omega)$ and $v \in L^{p^{\prime}(x)}(\Omega)$, we have

$$
\left|\int_{\Omega} u v d x\right| \leq\left(\frac{1}{p^{-}}+\frac{1}{\left(p^{\prime}\right)^{-}}\right)|u|_{p(x)}|v|_{p^{\prime}(x)} \leq 2|u|_{p(x)}|v|_{p^{\prime}(x)} .
$$

Proposition $2.2([11,18])$. Denote $\rho(u)=\int_{\Omega}|u(x)|^{p(x)} d x, \forall u \in L^{p(x)} \Omega$, then we have
i) $|u|_{p(x)}<1(=1 ;>1) \Leftrightarrow \rho(u)<1(=1 ;>1)$,
ii) $|u|_{p(x)}>1 \Longrightarrow|u|_{p(x)}^{p^{-}} \leq \rho(u) \leq|u|_{p(x)}^{p^{+}}$,
iii) $|u|_{p(x)}<1 \Longrightarrow|u|_{p(x)}^{p^{+}} \leq \rho(u) \leq|u|_{p(x)}^{p^{-}}$.

Proposition $2.3([11,18])$. If $u, u_{n} \in L^{p(x)}(\Omega), n=1,2, \ldots$, then the following statements are equivalent to each other:

(1) $\lim _{n \rightarrow \infty}\left|u_{n}-u\right|_{p(x)}=0$;

(2) $\lim _{n \rightarrow \infty} \rho\left(u_{n}-u\right)=0$

(3) $u_{n} \rightarrow u$ in measure in $\Omega$ and $\lim _{n \rightarrow \infty} \rho\left(u_{n}\right)=\rho(u)$.

Define the variable exponent Sobolev space $W^{1, p(x)}(\Omega)$ by

$$
W^{1, p(x)}(\Omega)=\left\{u \in L^{p(x)}(\Omega) ;|\nabla u| \in L^{p(x)}(\Omega)\right\}
$$

and it can be equipped with the norm

$$
\|u\|=|u|_{p(x)}+|\nabla u|_{p(x)}, \forall u \in W^{1, p(x)}(\Omega) .
$$

The space $W_{0}^{1, p(x)}(\Omega)$ is denoted by the closure of $C_{0}^{\infty}(\Omega)$ in $W^{1, p(x)}(\Omega)$. We will use $\|u\|=|\nabla u|_{p(x)}$ for $u \in W_{0}^{1, p(x)}(\Omega)$ in the following discussions.

Proposition $2.4([11,18])$. If $p^{-}>1$ and $p^{+}<\infty$, then the spaces $L^{p(x)}(\Omega)$, $L_{c(x)}^{p(x)}(\Omega), W^{1, p(x)}(\Omega)$ and $W_{0}^{1, p(x)}(\Omega)$ are separable and reflexive Banach spaces.

Given two Banach spaces $X$ and $Y$ the symbol $X \hookrightarrow Y$ means that $X$ is continuously embedded in $Y$ and also the symbol $X \hookrightarrow \hookrightarrow Y$ means that there is a compact embedding of $X$ in $Y$.

Proposition 2.5 ([11, 18]). (i) Assume that the boundary of $\Omega$ possesses the cone property and $p \in C(\bar{\Omega})$. If $q \in C(\bar{\Omega})$ and $1 \leq q(x)<p^{*}(x)$ for any $x \in \bar{\Omega}$, then $W^{1, p(x)}(\Omega) \hookrightarrow \hookrightarrow L^{q(x)}(\Omega)$.

(ii) If $p, q \in C(\bar{\Omega})$ and $p(x) \leq q(x) \leq p^{*}(x)$ for any $x \in \bar{\Omega}$, then $W^{1, p(x)}(\Omega)$ $\hookrightarrow L^{q(x)}(\Omega)$ and also there is a constant $c>0$ such that

$$
|u|_{q(x)} \leq c\|u\| \quad \forall u \in W_{0}^{1, p(x)}(\Omega) .
$$


Proposition $2.6([8])$. Let $p(x)$ and $q(x)$ be measurable functions such that $p(x) \in L^{\infty}(\Omega)$ and $1 \leq p(x) q(x) \leq \infty$ for a.e. $x \in \Omega$. Let $u \in L^{q(x)}(\Omega), u \neq 0$. Then

$$
\begin{gathered}
|u|_{p(x) q(x)} \leq 1 \Longrightarrow|u|_{p(x) q(x)}^{p^{+}} \leq\left.\left.|| u\right|^{p(x)}\right|_{q(x)} \leq|u|_{p(x) q(x)}^{p^{-}} \\
|u|_{p(x) q(x)} \geq 1 \Longrightarrow|u|_{p(x) q(x)}^{p^{-}} \leq\left.\left.|| u\right|^{p(x)}\right|_{q(x)} \leq|u|_{p(x) q(x)}^{p^{+}} .
\end{gathered}
$$

In particular, if $p(x)=p$ is constant, then

$$
\left.\left.|| u\right|^{p}\right|_{q(x)}=|u|_{p q(x)}^{p} .
$$

Theorem 2.7. Assume that the boundary of $\Omega$ possesses the cone property and $p \in C(\bar{\Omega})$. Suppose that $b \in L^{\beta(x)}(\Omega), b(x)>0$ for $x \in \Omega, \beta \in C(\bar{\Omega})$ and $\beta^{-}>1, \beta_{0}^{-} \leq \beta_{0}(x) \leq \beta_{0}^{+}\left(\frac{1}{\beta(x)}+\frac{1}{\beta_{0}(x)}=1\right)$. If $h \in C(\bar{\Omega})$ and

$$
1<h(x)<\frac{\beta(x)-1}{\beta(x)} p^{*}(x), \forall x \in \bar{\Omega}
$$

or

$$
1<\beta(x)<\frac{N p(x)}{N p(x)-h(x)(N-p(x))},
$$

then the embedding from $W^{1, p(x)}(\Omega)$ to $L_{b(x)}^{h(x)}(\Omega)$ is compact. Moreover, there is a constant $c_{5}>0$ such that the inequality

$$
\int_{\Omega} b(x)|u|^{h(x)} d x \leq c_{5}\left(\|u\|^{h^{-}}+\|u\|^{h^{+}}\right)
$$

holds.

Proof. We must remark that our proof of the embedding $W^{1, p(x)}(\Omega) \hookrightarrow \hookrightarrow$ $L_{b(x)}^{h(x)}(\Omega)$ is similar to Fan [10]. Let $u \in W^{1, p(x)}(\Omega)$ and set $r(x)=\frac{\beta(x)}{\beta(x)-1} h(x)$ $=\beta_{0}(x) h(x)$. Then (2.1) implies $r(x)<p^{*}(x)$. Hence, by Proposition 2.5 we have the embedding $W^{1, p(x)}(\Omega) \hookrightarrow \hookrightarrow L^{r(x)}(\Omega)$. So, for $u \in W^{1, p(x)}(\Omega)$, we have $|u|^{h(x)} \in L^{\beta_{0}(x)}(\Omega)$. By Proposition 2.1,

$$
\int_{\Omega} b(x)|u|^{h(x)} d x \leq\left.\left. c_{1}|b|_{\beta(x)}|| u\right|^{h(x)}\right|_{\beta_{0}(x)}<\infty .
$$

This implies that $W^{1, p(x)}(\Omega) \subset L_{b(x)}^{h(x)}(\Omega)$. Now let $\left\{u_{n}\right\} \subset W^{1, p(x)}(\Omega)$ and

$$
u_{n} \rightarrow 0 \text { (weakly) in } W^{1, p(x)}(\Omega) .
$$

Then, we have

$$
u_{n} \rightarrow 0 \text { (strongly) in } L^{r(x)}(\Omega) .
$$

So, it follows that $\left.\left.|| u_{n}\right|^{h(x)}\right|_{\beta_{0}(x)} \rightarrow 0$. Thus, we have

$$
\int_{\Omega} b(x)\left|u_{n}\right|^{h(x)} d x \leq\left.\left. c_{1}|b|_{\beta(x)}|| u_{n}\right|^{h(x)}\right|_{\beta_{0}(x)} \rightarrow 0,
$$


which implies $\left|u_{n}\right|_{(h(x), b(x))} \rightarrow 0$. Hence, we have the embedding $W^{1, p(x)}(\Omega) \hookrightarrow \hookrightarrow$ $L_{b(x)}^{h(x)}(\Omega)$.

Now let's show the inequality (2.2) holds. By the above inequity we know that

$$
\int_{\Omega} b(x)|u|^{h(x)} d x \leq\left.\left. c_{1}|b|_{\beta(x)}|| u\right|^{h(x)}\right|_{\beta_{0}(x)}<\infty .
$$

Since $h^{-} \leq h(x) \leq h^{+}$and $|u|^{h(x)} \leq|u|^{h^{-}}+|u|^{h^{+}}$it follows that

$$
\int_{\Omega} b(x)|u|^{h(x)} d x \leq \int_{\Omega} b(x)|u|^{h^{-}} d x+\int_{\Omega} b(x)|u|^{h^{+}} d x .
$$

Moreover, by Propositions 2.1, 2.2, 2.5, 2.6 and the condition $p(x)<h^{-} \beta_{0}(x) \leq$ $h^{+} \beta_{0}(x)<p^{*}(x)$, we have

$$
\int_{\Omega} b(x)|u|^{h^{-}} d x \leq\left.\left. c_{2}|b|_{\beta(x)}|| u\right|^{h^{-}}\right|_{\beta_{0}(x)}=c_{2}|b|_{\beta(x)}|u|_{h^{-} \beta_{0}(x)}^{h^{-}} \leq c_{3}\|u\|^{h^{-}} .
$$

Similarly, we can obtain

$$
\int_{\Omega} b(x)|u|^{h^{+}} d x \leq c_{4}\|u\|^{h^{+}} .
$$

As a result, from (2.3) and (2.4) it follows that

$$
\int_{\Omega} b(x)|u|^{h(x)} d x \leq c_{5}\left(\|u\|^{h^{-}}+\|u\|^{h^{+}}\right) .
$$

The proof is complete.

Theorem 2.8. Assume that the boundary of $\Omega$ possesses the cone property and $p \in C(\bar{\Omega})$. Suppose that $a \in L^{\alpha(x)}(\Omega), a(x)>0$ for $x \in \Omega, \alpha \in C(\bar{\Omega})$ and $\alpha^{-}>1, \alpha_{0}^{-} \leq \alpha_{0}(x) \leq \alpha_{0}^{+}\left(\frac{1}{\alpha(x)}+\frac{1}{\alpha_{0}(x)}=1\right)$. If $q \in C(\bar{\Omega}), p(x)<$ $\frac{\alpha(x)}{\alpha(x)-1} q(x)$ and

$$
1<q(x)<\frac{\alpha(x)-1}{\alpha(x)} p^{*}(x), \forall x \in \bar{\Omega}
$$

or

$$
\frac{N p(x)}{N p(x)-q(x)(N-p(x))}<\alpha(x)<\frac{p(x)}{p(x)-q(x)} \quad \text { with }
$$

then the embedding from $W^{1, p(x)}(\Omega)$ to $L_{a(x)}^{q(x)}(\Omega)$ is compact. Moreover, there is a constant $c_{7}>0$ such that the inequality

$$
\int_{\Omega} a(x)|u|^{q(x)} d x \leq c_{7}\left(\|u\|^{q^{-}}+\|u\|^{q^{+}}\right)
$$

holds. 
Proof. Let $u \in W^{1, p(x)}(\Omega)$. Set $m(x)=\frac{\alpha(x)}{\alpha(x)-1} q(x)=\alpha_{0}(x) q(x)$. Then $(2.5)$ implies $m(x)<p^{*}(x)$. Hence, by Proposition 2.5 there is the embedding $W^{1, p(x)}(\Omega) \hookrightarrow \hookrightarrow L^{m(x)}(\Omega)$. For $u \in W^{1, p(x)}(\Omega)$ we have $|u|^{q(x)} \in L^{\alpha_{0}(x)}(\Omega)$. By Proposition 2.1,

$$
\int_{\Omega} a(x)|u|^{q(x)} d x \leq\left.\left. c_{6}|a|_{\alpha(x)}|| u\right|^{q(x)}\right|_{\alpha_{0}(x)}<\infty .
$$

This implies that $W^{1, p(x)}(\Omega) \subset L_{a(x)}^{q(x)}(\Omega)$. Now let $\left\{u_{n}\right\} \subset W^{1, p(x)}(\Omega)$ and

$$
u_{n} \rightarrow 0 \text { in } W^{1, p(x)}(\Omega) .
$$

Then, we have

$$
u_{n} \rightarrow 0 \text { in } L^{m(x)}(\Omega) .
$$

So, it follows that $\left.\left.|| u_{n}\right|^{q(x)}\right|_{\alpha_{0}(x)} \rightarrow 0$. Thus, we have

$$
\int_{\Omega} a(x)|u|^{q(x)} d x \leq\left.\left. c_{6}|a|_{\alpha(x)}|| u\right|^{q(x)}\right|_{\alpha_{0}(x)} \rightarrow 0,
$$

which implies $\left|u_{n}\right|_{(q(x), a(x))} \rightarrow 0$. Hence, we have the embedding $W^{1, p(x)}(\Omega) \hookrightarrow \hookrightarrow$ $L_{a(x)}^{q(x)}(\Omega)$.

Now, let's show that the inequality (2.6) holds. By the above inequality we know that

$$
\int_{\Omega} a(x)|u|^{q(x)} d x \leq\left.\left. c_{6}|a|_{\alpha(x)}|| u\right|^{q(x)}\right|_{\alpha_{0}(x)}<\infty .
$$

Considering the condition $p(x)<q^{-} \alpha_{0}(x) \leq q^{+} \alpha_{0}(x)<p^{*}(x)$ and applying the similar steps as we did in proof of Theorem 2.7 , we have

$$
\int_{\Omega} a(x)|u|^{q(x)} d x \leq c_{7}\left(\|u\|^{q^{-}}+\|u\|^{q^{+}}\right) .
$$

The proof is complete.

Proposition 2.9. Assume that the conditions of Theorem 2.7 and Theorem 2.8 hold, respectively. Let $u \in W^{1, p(x)}(\Omega)$, then there are positive constants $c_{8}, c_{9}$, $c_{10}, c_{11}>0$ such that the following inequalities hold

i)

$$
\int_{\Omega} b(x)|u|^{h(x)} d x \leq \begin{cases}c_{8}\|u\|^{h^{+}} & \text {if }\|u\|>1 \\ c_{9}\|u\|^{h^{-}} & \text {if }\|u\|<1\end{cases}
$$

ii)

$$
\int_{\Omega} a(x)|u|^{q(x)} d x \leq \begin{cases}c_{10}\|u\|^{q^{+}} & \text {if }\|u\|>1 \\ c_{11}\|u\|^{q^{-}} & \text {if }\|u\|<1 .\end{cases}
$$


Proof. It follows immediately from the conclusions of Theorem 2.7 and Theorem 2.8, respectively.

\section{The main results}

Let $J \in C^{1}(X, \mathbb{R})$ be the Euler functional associated with an elliptic problem on Banach space $X$. If $J$ is bounded below and has a minimizer on $X$, then this minimizer is a critical point of $J$. Hence, it is a solution of the corresponding elliptic problem. However, in many problems $J$ is not bounded below on the whole space $X$, but is bounded below on an appropriate subset of $X$, and minimizer on this set (if it exists) may give rise to solutions of the corresponding elliptic problem. A good candidate for an appropriate subset of $X$ is the Nehari manifold.

If we consider our problem $\left(E_{\lambda}\right)$, then, the corresponding Euler functional is defined by

$$
J_{\lambda}(u)=\int_{\Omega} \frac{1}{p(x)}|\nabla u|^{p(x)} d x-\lambda \int_{\Omega} \frac{1}{q(x)} a(x)|u|^{q(x)} d x-\int_{\Omega} \frac{1}{h(x)} b(x)|u|^{h(x)} d x .
$$

Then, by Theorems 2.7, 2.8, and Proposition 2.2, we have

$$
\begin{aligned}
J_{\lambda}(u) & \geq \frac{1}{p^{+}} \int_{\Omega}|\nabla u|^{p(x)} d x-\frac{\lambda}{q^{-}} \int_{\Omega} a(x)|u|^{q(x)} d x-\frac{1}{h^{-}} \int_{\Omega} b(x)|u|^{h(x)} d x, \\
& \geq \frac{1}{p^{+}}\|u\|^{p^{-}}-\frac{\lambda}{q^{-}} c_{7}\left(\|u\|^{q^{-}}+\|u\|^{q^{+}}\right)-\frac{1}{h^{-}} c_{5}\left(\|u\|^{h^{-}}+\|u\|^{h^{+}}\right) .
\end{aligned}
$$

Since $q^{+}<p^{-} \leq p^{+}<h^{-} \leq h^{+}$, this shows $J_{\lambda}$ is not bounded below on whole $W_{0}^{1, p(x)}(\Omega)$. However, we shall show it is bounded on the Nehari manifold $M_{\lambda}(\Omega)$ which is given by

$$
M_{\lambda}(\Omega)=\left\{u \in W_{0}^{1, p(x)}(\Omega) \backslash\{0\}:\left\langle J_{\lambda}^{\prime}(u), u\right\rangle=0\right\},
$$

where $\langle.,$.$\rangle denotes the usual duality between W_{0}^{1, p(x)}(\Omega)$ and $W^{-1, p^{\prime}(x)}(\Omega)$. It is clear that all critical points of $J_{\lambda}$ must lie on $M_{\lambda}(\Omega)$ and local minimizers on $M_{\lambda}(\Omega)$ are usually critical points of $J_{\lambda}$.

Thus, $u \in M_{\lambda}(\Omega)$ if and only if

$$
\begin{aligned}
I_{\lambda}(u):=\left\langle J_{\lambda}^{\prime}(u), u\right\rangle & =\int_{\Omega}|\nabla u|^{p(x)} d x-\lambda \int_{\Omega} a(x)|u|^{q(x)} d x-\int_{\Omega} b(x)|u|^{h(x)} d x \\
& =0 .
\end{aligned}
$$


Then for $u \in M_{\lambda}(\Omega)$, we have

$$
\begin{aligned}
\left\langle I_{\lambda}^{\prime}(u), u\right\rangle= & \int_{\Omega} p(x)|\nabla u|^{p(x)} d x-\lambda \int_{\Omega} q(x) a(x)|u|^{q(x)} d x \\
& \quad-\int_{\Omega} h(x) b(x)|u|^{h(x)} d x \\
\leq & \left(p^{+}-q^{-}\right) \lambda \int_{\Omega} a(x)|u|^{q(x)} d x+\left(p^{+}-h^{-}\right) \int_{\Omega} b(x)|u|^{h(x)} d x .
\end{aligned}
$$

Now, we split $M_{\lambda}(\Omega)$ into three parts:

$$
\begin{aligned}
& M_{\lambda}^{+}=\left\{u \in M_{\lambda}(\Omega):\left\langle I_{\lambda}^{\prime}(u), u\right\rangle>0\right\}, \\
& M_{\lambda}^{-}=\left\{u \in M_{\lambda}(\Omega):\left\langle I_{\lambda}^{\prime}(u), u\right\rangle<0\right\}, \\
& M_{\lambda}^{0}=\left\{u \in M_{\lambda}(\Omega):\left\langle I_{\lambda}^{\prime}(u), u\right\rangle=0\right\} .
\end{aligned}
$$

Theorem 3.1. Suppose that $u_{0}$ is a local maximum or minimum for $J_{\lambda}$ on $M_{\lambda}(\Omega)$. If $u_{0} \notin M_{\lambda}^{0}(\Omega)$, then $u_{0}$ is a critical point of $J_{\lambda}$.

Proof. The proof of Theorem 3.1 can be obtained directly from the following lemmas (particularly Lemma 3.3).

Lemma 3.2. The energy functional $J_{\lambda}$ is coercive and bounded below on $M_{\lambda}(\Omega)$.

Proof. Let $u \in M_{\lambda}(\Omega)$ and $\|u\|>1$. Then using (3.1), Propositions 2.2 and 2.9 , we have

$$
\begin{aligned}
J_{\lambda}(u)= & \int_{\Omega} \frac{1}{p(x)}|\nabla u|^{p(x)} d x-\lambda \int_{\Omega} \frac{1}{q(x)} a(x)|u|^{q(x)} d x-\int_{\Omega} \frac{1}{h(x)} b(x)|u|^{h(x)} d x \\
\geq & \frac{1}{p^{+}} \int_{\Omega}|\nabla u|^{p(x)} d x-\frac{\lambda}{q^{-}} \int_{\Omega} a(x)|u|^{q(x)} d x \\
& -\frac{1}{h^{-}}\left(\int_{\Omega}|\nabla u|^{p(x)} d x-\lambda \int_{\Omega} a(x)|u|^{q(x)} d x\right) \\
\geq & \left(\frac{1}{p^{+}}-\frac{1}{h^{-}}\right) \int_{\Omega}|\nabla u|^{p(x)} d x+\lambda\left(\frac{1}{h^{-}}-\frac{1}{q^{-}}\right) \int_{\Omega} a(x)|u|^{q(x)} d x \\
\geq & \left(\frac{h^{-}-p^{+}}{h^{-} p^{+}}\right)\|u\|^{p^{-}}-c_{10} \lambda\left(\frac{h^{-}-q^{-}}{h^{-} q^{-}}\right)\|u\|^{q^{+}} .
\end{aligned}
$$

Since, $p^{-}>q^{+}$so, $J_{\lambda}(u) \rightarrow \infty$ as $\|u\| \rightarrow \infty$. This implies $J_{\lambda}$ is coercive and bounded below on $M_{\lambda}(\Omega)$.

Lemma 3.3. There exists $\lambda_{1}>0$ such that for $0<\lambda<\lambda_{1}$ we have $M_{\lambda}^{0}(\Omega)=$ $\varnothing$. 
Proof. Suppose otherwise, that is, $M_{\lambda}^{0}(\Omega) \neq \varnothing$ for all $\lambda \in \mathbb{R} \backslash\{0\}$. Let $u \in$ $M_{\lambda}^{0}(\Omega)$ such that $\|u\|>1$. Then using (3.1), (2.4) and definition of $M_{\lambda}^{0}(\Omega)$, we have

$$
\begin{aligned}
0= & \left\langle I_{\lambda}^{\prime}(u), u\right\rangle \\
= & \int_{\Omega} p(x)|\nabla u|^{p(x)} d x-\lambda \int_{\Omega} q(x) a(x)|u|^{q(x)} d x-\int_{\Omega} h(x) b(x)|u|^{h(x)} d x \\
\geq & p^{-} \int_{\Omega}|\nabla u|^{p(x)} d x-q^{+}\left(\int_{\Omega}|\nabla u|^{p(x)} d x-\int_{\Omega} b(x)|u|^{h(x)} d x\right) \\
& -h^{+} \int_{\Omega} b(x)|u|^{h(x)} d x \\
\geq & \left(p^{-}-q^{+}\right) \int_{\Omega}|\nabla u|^{p(x)} d x+\left(q^{+}-h^{+}\right) \int_{\Omega} b(x)|u|^{h(x)} d x .
\end{aligned}
$$

By Proposition 2.9,

$$
0 \geq\left(p^{-}-q^{+}\right)\|u\|^{p^{-}}+c_{8}\left(q^{+}-h^{+}\right)\|u\|^{h^{+}},
$$

$$
\|u\| \geq c_{12}\left(\frac{p^{-}-q^{+}}{h^{+}-q^{+}}\right)^{\frac{1}{h^{+}-p^{-}}} .
$$

Similarly,

$$
\begin{aligned}
0 & =\left\langle I_{\lambda}^{\prime}(u), u\right\rangle \\
\leq & p^{+} \int_{\Omega}|\nabla u|^{p(x)} d x-\lambda q^{-} \int_{\Omega} a(x)|u|^{q(x)} d x-h^{-} \int_{\Omega} b(x)|u|^{h(x)} d x \\
\leq & p^{+} \int_{\Omega}|\nabla u|^{p(x)} d x-\lambda q^{-} \int_{\Omega} a(x)|u|^{q(x)} d x \\
& -h^{-}\left(\int_{\Omega}|\nabla u|^{p(x)} d x-\lambda \int_{\Omega} a(x)|u|^{q(x)} d x\right) .
\end{aligned}
$$

By Proposition 2.9,

$$
\begin{gathered}
0 \leq\left(p^{+}-h^{-}\right)\|u\|^{p^{-}}+\lambda c_{10}\left(h^{-}-q^{-}\right)\|u\|^{q^{+}}, \\
\|u\| \leq c_{13}\left(\lambda \frac{h^{-}-q^{-}}{h^{-}-p^{+}}\right)^{\frac{1}{p^{-}-q^{+}}} .
\end{gathered}
$$


If $\lambda$ is sufficiently small (e.g. $\lambda=\left(\frac{h^{-}-p^{+}}{h^{-}-q^{-}}\right)\left(\frac{p^{-}-q^{+}}{h^{+}-q^{+}}\right)^{\frac{p^{-}-q^{+}}{h^{+}-p^{-}}}$), then from (3.2) and (3.3) we get $\|u\|<1$ which contradicts with our assumption. Hence, we conclude $M_{\lambda}^{0}(\Omega)=\varnothing$.

By Lemma 3.3, for $0<\lambda<\lambda_{1}$, we can write $M_{\lambda}(\Omega)=M_{\lambda}^{+}(\Omega) \cup M_{\lambda}^{-}(\Omega)$. Therefore, we can let

$$
\alpha_{\lambda}^{+}=\inf _{u \in M_{\lambda}^{+}(\Omega)} J_{\lambda}(u) \text { and } \alpha_{\lambda}^{-}=\inf _{u \in M_{\lambda}^{-}(\Omega)} J_{\lambda}(u) .
$$

Lemma 3.4. If $0<\lambda<\lambda_{1}$, then for all $u \in M_{\lambda}^{+}(\Omega), J_{\lambda}(u)<0$.

Proof. Let $u \in M_{\lambda}^{+}(\Omega)$. By definition of $J_{\lambda}(u)$, we can write

(3.4) $J_{\lambda}(u) \leq \frac{1}{p^{-}} \int_{\Omega}|\nabla u|^{p(x)} d x-\frac{\lambda}{q^{+}} \int_{\Omega} a(x)|u|^{q(x)} d x-\frac{1}{h^{+}} \int_{\Omega} b(x)|u|^{h(x)} d x$.

Since $u \in M_{\lambda}^{+}(\Omega)$, we have

$$
p^{+} \int_{\Omega}|\nabla u|^{p(x)} d x-\lambda q^{-} \int_{\Omega} a(x)|u|^{q(x)} d x-h^{-} \int_{\Omega} b(x)|u|^{h(x)} d x>0 .
$$

Now, if we multiply (3.1) by $\left(-q^{-}\right)$and add with (3.5), we get

$$
\int_{\Omega} b(x)|u|^{h(x)} d x<\frac{p^{+}-q^{-}}{h^{-}-q^{-}} \int_{\Omega}|\nabla u|^{p(x)} d x .
$$

Moreover, using (3.1) together with (3.4)

$$
J_{\lambda}(u) \leq\left(\frac{1}{p^{-}}-\frac{1}{q^{+}}\right) \int_{\Omega}|\nabla u|^{p(x)} d x+\left(\frac{1}{q^{+}}-\frac{1}{h^{+}}\right) \int_{\Omega} b(x)|u|^{h(x)} d x,
$$

and applying (3.6) in (3.7), it follows

$$
J_{\lambda}(u)<-\frac{\left(p^{-}-q^{+}\right)\left(h^{+}-p^{-}\right)}{h^{+} p^{-} q^{+}}\|u\|^{p^{-}}<0 .
$$

Hence, we have $\alpha_{\lambda}^{+}=\inf _{u \in M_{\lambda}^{+}(\Omega)} J_{\lambda}(u)<0$.

Theorem 3.5. If $0<\lambda<\lambda_{1}$, there exists a minimizer of $J_{\lambda}$ on $M_{\lambda}^{+}(\Omega)$.

Proof. Since $J_{\lambda}$ is bounded below on $M_{\lambda}(\Omega)$ and so on $M_{\lambda}^{+}(\Omega)$. Then, there exits a minimizing sequence $\left\{u_{n}^{+}\right\} \subseteq M_{\lambda}^{+}(\Omega)$ such that

$$
\lim _{n \rightarrow \infty} J_{\lambda}\left(u_{n}^{+}\right)=\inf _{u \in M_{\lambda}^{+}(\Omega)} J_{\lambda}(u)=\alpha_{\lambda}^{+}<0 .
$$

Since $J_{\lambda}$ is coercive, $u_{n}^{+}$is bounded in $W_{0}^{1, p(x)}(\Omega)$. Thus, we may assume that, without loss of generality, $u_{n}^{+} \rightarrow u_{0}^{+}$in $W_{0}^{1, p(x)}(\Omega)$ and by the compact embeddings we have

$$
u_{n}^{+} \rightarrow u_{0}^{+} \text {in } L_{a(x)}^{q(x)}(\Omega),
$$


and

$$
u_{n}^{+} \rightarrow u_{0}^{+} \text {in } L_{b(x)}^{h(x)}(\Omega) .
$$

Now, we shall prove $u_{n}^{+} \rightarrow u_{0}^{+}$in $W_{0}^{1, p(x)}(\Omega)$. Otherwise, suppose $u_{n}^{+} \nrightarrow u_{0}^{+}$in $W_{0}^{1, p(x)}(\Omega)$. Then

$$
\int_{\Omega}\left|\nabla u_{0}^{+}\right|^{p(x)} d x<\lim _{n \rightarrow \infty} \inf \int_{\Omega}\left|\nabla u_{n}^{+}\right|^{p(x)} d x .
$$

Moreover, by the compact embeddings we have

$$
\begin{aligned}
& \int_{\Omega} a(x)\left|u_{0}^{+}\right|^{q(x)} d x=\lim _{n \rightarrow \infty} \inf \int_{\Omega} a(x)\left|u_{n}^{+}\right|^{q(x)} d x, \\
& \int_{\Omega} b(x)\left|u_{0}^{+}\right|^{h(x)} d x=\lim _{n \rightarrow \infty} \inf \int_{\Omega} b(x)\left|u_{n}^{+}\right|^{h(x)} d x .
\end{aligned}
$$

Using the fact that $\left\langle J_{\lambda}^{\prime}\left(u_{n}^{+}\right), u_{n}^{+}\right\rangle=0$ and Theorem 2.8, we can write the followings

$$
\begin{aligned}
J_{\lambda}\left(u_{n}^{+}\right) \geq & \left(\frac{1}{p^{+}}-\frac{1}{h^{-}}\right) \int_{\Omega}\left|\nabla u_{n}^{+}\right|^{p(x)} d x+\lambda\left(\frac{1}{h^{-}}-\frac{1}{q^{-}}\right) \int_{\Omega} a(x)\left|u_{n}^{+}\right|^{q(x)} d x \\
\lim _{n \rightarrow \infty} J_{\lambda}\left(u_{n}^{+}\right) \geq & \left(\frac{1}{p^{+}}-\frac{1}{h^{-}}\right) \lim _{n \rightarrow \infty} \int_{\Omega}\left|\nabla u_{n}^{+}\right|^{p(x)} d x \\
& +\lambda\left(\frac{1}{h^{-}}-\frac{1}{q^{-}}\right) \lim _{n \rightarrow \infty} \int_{\Omega} a(x)\left|u_{n}^{+}\right|^{q(x)} d x \\
\alpha_{\lambda}^{+}= & \inf _{u \in M_{\lambda}^{+}} J_{\lambda}(u) \\
> & \left(\frac{1}{p^{+}}-\frac{1}{h^{-}}\right)\left\|u_{0}^{+}\right\|^{p^{-}}+c_{7} \lambda\left(\frac{1}{h^{-}}-\frac{1}{q^{-}}\right)\left(\left\|u_{0}^{+}\right\|^{q^{-}}+\left\|u_{0}^{+}\right\|^{q^{+}}\right)
\end{aligned}
$$

since $p^{-}>q^{+}$, for $\left\|u_{0}^{+}\right\|>1$, we have

$$
\alpha_{\lambda}^{+}=\inf _{u \in M_{\lambda}^{+}} J_{\lambda}(u)>0 .
$$

However, in Lemma 3.4 it was showed that for any $u \in M_{\lambda}^{+}(\Omega), J_{\lambda}(u)<0$. So, this is a contradiction. Hence, $u_{n}^{+} \rightarrow u_{0}^{+}$in $W_{0}^{1, p(x)}(\Omega)$ and

$$
J_{\lambda}\left(u_{0}^{+}\right)=\lim _{n \rightarrow \infty} J_{\lambda}\left(u_{n}^{+}\right)=\inf _{u \in M_{\lambda}^{+}(\Omega)} J_{\lambda}(u) .
$$

Thus, $u_{0}^{+}$is a minimizer for $J_{\lambda}$ on $M_{\lambda}^{+}(\Omega)$.

Lemma 3.6. If $0<\lambda<\lambda_{1}$, then for all $u \in M_{\lambda}^{-}(\Omega), J_{\lambda}(u)>0$. 
Proof. Let $u \in M_{\lambda}(\Omega)$. By definition of $J_{\lambda}(u)$ and (3.1), we have

$$
J_{\lambda}(u) \geq \frac{1}{p^{+}} \int_{\Omega}|\nabla u|^{p(x)} d x-\frac{\lambda}{q^{-}} \int_{\Omega} a(x)|u|^{q(x)} d x-\frac{1}{h^{-}} \int_{\Omega} b(x)|u|^{h(x)} d x,
$$

and

$$
\int_{\Omega} b(x)|u|^{h(x)} d x=\int_{\Omega}|\nabla u|^{p(x)} d x-\lambda \int_{\Omega} a(x)|u|^{q(x)} d x .
$$

Using the two expressions above, it follows

$$
\begin{aligned}
J_{\lambda}(u) \geq \frac{1}{p^{+}} \int_{\Omega}|\nabla u|^{p(x)} d x-\frac{\lambda}{q^{-}} \int_{\Omega} a(x)|u|^{q(x)} d x \\
\quad-\frac{1}{h^{-}}\left(\int_{\Omega}|\nabla u|^{p(x)} d x-\lambda \int_{\Omega} a(x)|u|^{q(x)} d x\right) \\
\geq\left(\frac{1}{p^{+}}-\frac{1}{h^{-}}\right) \int_{\Omega}|\nabla u|^{p(x)} d x+\lambda\left(\frac{1}{h^{-}}-\frac{1}{q^{-}}\right) \int_{\Omega} a(x)|u|^{q(x)} d x .
\end{aligned}
$$

By Propositions 2.2, 2.9, and the condition $p^{-}>q^{+}$, it follows

$$
\begin{aligned}
J_{\lambda}(u) & \geq\left(\frac{1}{p^{+}}-\frac{1}{h^{-}}\right)\|u\|^{p^{-}}+c_{10} \lambda\left(\frac{1}{h^{-}}-\frac{1}{q^{-}}\right)\|u\|^{q+} \\
& \geq\left(\frac{h^{-}-p^{+}}{p^{+} h^{-}}+c_{10} \lambda \frac{q^{-}-h^{-}}{q^{-} h^{-}}\right)\|u\|^{p^{-}} .
\end{aligned}
$$

So, if we choose $\lambda<\frac{q^{-}\left(h^{-}-p^{+}\right)}{c_{10} p^{+}\left(h^{-}-q^{-}\right)}$, we get $J_{\lambda}(u)>0$. Moreover, if we consider the facts $M_{\lambda}(\Omega)=M_{\lambda}^{+}(\Omega) \cup M_{\lambda}^{-}(\Omega)$ (see Lemma 3.3), $M_{\lambda}^{+}(\Omega) \cap M_{\lambda}^{-}(\Omega)=\varnothing$, and Lemma 3.4, we must have $u \in M_{\lambda}^{-}(\Omega)$.

Theorem 3.7. If $0<\lambda<\lambda_{1}$, there exists a minimizer of $J_{\lambda}$ on $M_{\lambda}^{-}(\Omega)$.

Proof. Since $J_{\lambda}$ is bounded below on $M_{\lambda}(\Omega)$ and so on $M_{\lambda}^{-}(\Omega)$, then there exits a minimizing sequence $\left\{u_{n}^{-}\right\} \subseteq M_{\lambda}^{-}(\Omega)$ such that

$$
\lim _{n \rightarrow \infty} J_{\lambda}\left(u_{n}^{-}\right)=\inf _{u \in M_{\lambda}^{-}(\Omega)} J_{\lambda}(u)=\alpha_{\lambda}^{-}>0 .
$$

Since $J_{\lambda}$ is coercive, $u_{n}^{-}$is bounded in $W_{0}^{1, p(x)}(\Omega)$. Thus, we may assume that, without loss of generality, $u_{n}^{-} \rightarrow u_{0}^{-}$in $W_{0}^{1, p(x)}(\Omega)$ and by the compact embeddings we have

$$
u_{n}^{-} \rightarrow u_{0}^{-} \text {in } L_{a(x)}^{q(x)}(\Omega),
$$

and

$$
u_{n}^{-} \rightarrow u_{0}^{-} \text {in } L_{b(x)}^{h(x)}(\Omega)
$$


Moreover, if $u_{0}^{-} \in M_{\lambda}^{-}(\Omega)$, then there is a constant $t>0$ such that $t u_{0}^{-}$ $\in M_{\lambda}^{-}(\Omega)$ and $J_{\lambda}\left(u_{0}^{-}\right) \geq J_{\lambda}\left(t u_{0}^{-}\right)$. Indeed, since

$I_{\lambda}^{\prime}(u)=\int_{\Omega} p(x)|\nabla u|^{p(x)} d x-\lambda \int_{\Omega} q(x) a(x)|u|^{q(x)} d x-\int_{\Omega} h(x) b(x)|u|^{h(x)} d x$,

then,

$$
\begin{aligned}
I_{\lambda}^{\prime}\left(t u_{0}^{-}\right) & =\int_{\Omega} p(x)\left|\nabla t u_{0}^{-}\right|^{p(x)} d x-\lambda \int_{\Omega} q(x) a(x)\left|t u_{0}^{-}\right|^{q(x)} d x-\int_{\Omega} h(x) b(x)\left|t u_{0}^{-}\right|^{h(x)} d x \\
& \leq t^{p^{+}} p^{+} \int_{\Omega}\left|\nabla u_{0}^{-}\right|^{p(x)} d x-\lambda t^{q^{-}} q^{-} \int_{\Omega} a(x)\left|u_{0}^{-}\right|^{q(x)} d x-t^{h^{-}} h^{-} \int_{\Omega} b(x)\left|u_{0}^{-}\right|^{h(x)} d x .
\end{aligned}
$$

Since $q^{-}<p^{+}<h^{-}$, and by the assumptions on $a$ and $b$, it follows $I_{\lambda}^{\prime}\left(t u_{0}^{-}\right)<0$. Hence, by the definition of $M_{\lambda}^{-}(\Omega), t u_{0}^{-} \in M_{\lambda}^{-}(\Omega)$.

Now, we shall show $u_{n}^{-} \rightarrow u_{0}^{-}$in $W_{0}^{1, p(x)}(\Omega)$. Otherwise, suppose $u_{n}^{-} \nrightarrow u_{0}^{-}$ in $W_{0}^{1, p(x)}(\Omega)$. Then using the fact that

$$
\int_{\Omega}\left|\nabla u_{0}^{-}\right|^{p(x)} d x<\lim _{n \rightarrow \infty} \inf \int_{\Omega}\left|\nabla u_{n}^{-}\right|^{p(x)} d x
$$

we have,

$$
\begin{aligned}
J_{\lambda}\left(t u_{0}^{-}\right) & \leq \frac{t^{p^{+}}}{p^{-}} \int_{\Omega}\left|\nabla u_{0}^{-}\right|^{p(x)} d x-\lambda \frac{t^{q^{-}}}{q^{+}} \int_{\Omega} a(x)\left|u_{0}^{-}\right|^{q(x)} d x-\frac{t^{h^{-}}}{h^{+}} \int_{\Omega} b(x)\left|u_{0}^{-}\right|^{h(x)} d x \\
& <\lim _{n \rightarrow \infty}\left[\frac{t^{p^{+}}}{p^{-}} \int_{\Omega}\left|\nabla u_{n}^{-}\right|^{p(x)} d x-\lambda \frac{t^{q^{-}}}{q^{+}} \int_{\Omega} a(x)\left|u_{n}^{-}\right|^{q(x)} d x-\frac{t^{h^{-}}}{h^{+}} \int_{\Omega} b(x)\left|u_{n}^{-}\right|^{h(x)} d x\right] \\
& \leq \lim _{n \rightarrow \infty} J_{\lambda}\left(t u_{n}^{-}\right) \leq \lim _{n \rightarrow \infty} J_{\lambda}\left(u_{n}^{-}\right)=\inf _{u \in M_{\lambda}^{-}(\Omega)} J_{\lambda}(u)=\alpha_{\lambda}^{-} .
\end{aligned}
$$

This implies that $J_{\lambda}\left(t u_{0}^{-}\right)<\inf _{u \in M_{\lambda}^{-}(\Omega)} J_{\lambda}(u)=\alpha_{\lambda}^{-}$, which is a contradiction. Hence, $u_{n}^{-} \rightarrow u_{0}^{-}$in $W_{0}^{1, p(x)}(\Omega)$ and so

$$
J_{\lambda}\left(u_{0}^{-}\right)=\lim _{n \rightarrow \infty} J_{\lambda}\left(u_{n}^{-}\right)=\inf _{u \in M_{\lambda}^{-}(\Omega)} J_{\lambda}(u) .
$$

Thus, $u_{0}^{-}$is a minimizer for $J_{\lambda}$ on $M_{\lambda}^{-}(\Omega)$.

Corollary 3.8. By Theorems 3.5 and 3.7, we conclude that there exists $u_{0}^{+} \in$ $M_{\lambda}^{+}(\Omega)$ and $u_{0}^{-} \in M_{\lambda}^{-}(\Omega)$ such that $J_{\lambda}\left(u_{0}^{+}\right)=\inf _{u \in M_{\lambda}^{+}(\Omega)} J_{\lambda}(u)$ and $J_{\lambda}\left(u_{0}^{-}\right)=$ $\inf _{u \in M_{\lambda}^{-}(\Omega)} J_{\lambda}(u)$. Moreover, since $J_{\lambda}\left(u_{0}^{ \pm}\right)=J_{\lambda}\left(\left|u_{0}^{ \pm}\right|\right)$and $\left|u_{0}^{ \pm}\right| \in M_{\lambda}^{ \pm}(\Omega)$, we may assume $u_{0}^{ \pm} \geq 0$. By Theorem 3.1, $u_{0}^{ \pm}$are critical points of $J_{\lambda}$ on $W_{0}^{1, p(x)}(\Omega)$ and hence are weak solutions (and so by standard regularity results classical 
solutions) of $\left(E_{\lambda}\right)$. Finally, by the Harnack inequality due to [25, 27], we obtain that $u_{0}^{ \pm}$are positive solutions of $\left(E_{\lambda}\right)$.

\section{References}

[1] E. Acerbi and G. Mingione, Regularity results for stationary electro-rheological fluids, Arch. Ration. Mech. Anal. 164 (2002), no. 3, 213-259.

[2] G. A. Afrouzi, S. Mahdavi, and Z. Naghizadeh, The Nehari manifold for p-Laplacian equation with Dirichlet boundary condition, Nonlinear Anal. Model. Control 12 (2007), no. 2, 143-155.

[3] A. Ambrosetti, H. Brezis and G. Cerami, Combined effects of concave and convex nonlinearities in some elliptic problems, J. Funct. Anal. 122 (1994), no. 2, 519-543.

[4] K. J. Brown and Y. Zhang, The Nehari manifold for a semilinear elliptic equation with a sign-changing weight function, J. Differential Equations 193 (2003), no. 2, 481-499.

[5] O. M. Buhrii and R. A. Mashiyev, Uniqueness of solutions of the parabolic variation inequality with variable exponent of nonlinearity, Nonlinear Anal. 10 (2009), 2325-2331.

[6] J. Chabrowski and Y. Fu, Existence of solutions for $p(x)$-Laplacian problems on a bounded domain, J. Math. Anal. Appl. 306 (2005), no. 2, 604-618.

[7] L. Diening, Theoretical and Numerical Results for Electrorheological Fluids, Ph. D. thesis, University of Frieburg, Germany, 2002.

[8] D. Edmunds and J. Rakosnik, Sobolev embeddings with variable exponent, Studia Math. 143 (2000), no. 3, 267-293.

[9] A. El Hamidi, Existence results to elliptic systems with nonstandard growth conditions, J. Math. Anal. Appl. 300 (2004), no. 1, 30-42.

[10] X. L. Fan, Solutions for $p(x)$-Laplacian Dirichlet problems with singular coefficients, J. Math. Anal. Appl. 312 (2005), no. 2, 464-477.

[11] X. L. Fan, J. S. Shen, and D. Zhao, Sobolev embedding theorems for spaces $W^{k, p(x)}(\Omega)$, J. Math. Anal. Appl. 262 (2001), no. 2, 749-760.

[12] X. L. Fan and D. Zhao, On the spaces $L^{p(x)}(\Omega)$ and $W^{m, p(x)}(\Omega)$, J. Math. Anal. Appl. 263 (2001), no. 2, 424-446.

[13] X. L. Fan and Q. H. Zhang, Existence of solutions for $p(x)$-Laplacian Dirichlet problem, Nonlinear Anal. 52 (2003), no. 8, 1843-1852.

[14] X. L. Fan, Q. Zhang, and D. Zhao, Eigenvalues of $p(x)$-Laplacian Dirichlet problem, J. Math. Anal. Appl. 302 (2005), no. 2, 306-317.

[15] T. C. Halsey, Electrorheological fluids, Science 258 (1992), 761-766.

[16] P. Harjulehto, P. Hästö, M. Koskenoja, and S. Varonen, The Dirichlet energy integral and variable exponent Sobolev spaces with zero boundary values, Potential Anal. 25 (2006), no. 3, 205-222.

[17] P. Hästö, The $p(x)$-Laplacian and applications, J. Anal. 15 (2007), 53-62.

[18] O. Kovăčik and J. Răkosnik, On spaces $L^{p(x)}$ and $W^{k, p(x)}$, Czechoslovak Math. J. 41(116) (1991), no. 4, 592-618.

[19] R. A. Mashiyev, Some properties of variable Sobolev capacity, Taiwanese J. Math. 12 (2008), no. 3, 671-678.

[20] M. Mihăilescu, Existence and multiplicity of solutions for an elliptic equation with $p(x)$ growth conditions, Glasg. Math. J. 48 (2006), no. 3, 411-418.

[21] M. Mihăilescu and V. Rădulescu, A multiplicity result for a nonlinear degenerate problem arising in the theory of electrorheological fluids, Proc. R. Soc. Lond. Ser. A Math. Phys. Eng. Sci. 462 (2006), no. 2073, 2625-2641.

[22] _ On a nonhomogeneous quasilinear eigenvalue problem in Sobolev spaces with variable exponent, Proc. Amer. Math. Soc. 135 (2007), no. 9, 2929-2937. 
[23] S. Ogras, R. A. Mashiyev, M. Avci, and Z. Yucedag, Existence of solutions for a class of elliptic systems in $\mathbb{R}^{N}$ involving the $(p(x), q(x))$-Laplacian, J. Inequal. Appl. 2008 (2008), Art. Id 612938, 16 pp.

[24] M. Růžička, Electrorheological Fluids: modeling and mathematical theory, Springer Lecture Notes in Math. Vol. 1748, Springer Verlag, Berlin, Heidelberg, New York, 2000.

[25] N. S. Trudinger, On Harnack type inequalities and their application to quasilinear elliptic equations, Comm. Pure Appl. Math. 20 (1967), 721-747.

[26] T. F. Wu, Multiplicity of positive solution of p-Laplacian problems with sign-changing weight functions, Int. J. Math. Anal. (Ruse) 1 (2007), no. 9-12, 557-563.

[27] X. Zhang and X. Liu, The local boundedness and Harnack inequality of p(x)-Laplace equation, J. Math. Anal. Appl. 332 (2007), no. 1, 209-218.

[28] V. V. Zhikov, Averaging of functionals of the calculus of variations and elasticity theory, (russian) Izv. Akad. Nauk SSSR Ser. Mat. 50 (1986), no. 4, 675-710, 877.

Rabil A. MashiyeV

Department of Mathematics

Faculty of Science and Arts

DiCLE UNIVERSITY

21280-Diyarbakir, Turkey

E-mail address: mrabil@dicle.edu.tr

SEZAi OGRAs

Department of Mathematics

Faculty of Science And Arts

DiCLE UNIVERSITY

21280-DiYARBAKIR, TURKEY

E-mail address: seazio@dicle.edu.tr

ZEHRA YuCEDAG

Department of Mathematics

Faculty of Science And ARts

DiClE UNIVERSITY

21280-DiYARBAKIR, TURKEY

E-mail address: zehra@dicle.edu.tr

Mustafa Avci

Department of Mathematics

Faculty of Science and Arts

DiClE UNIVERSITY

21280-DiYARBAKIR, TURKEY

E-mail address: mustafaxavci@hotmail.com 